\title{
Newton's Method Obeys Benford's Law
}

\section{Arno Berger and Theodore P. Hill}

1. INTRODUCTION. In scientific calculations using digital computers and floatingpoint arithmetic, roundoff errors are inevitable, even with the most elementary of functions. For example, a very simple, hypothetical computer with only one decimal point precision, equipped with the IEEE Standard "Unbiased" Rounding, approximates the function $f(x)=x^{2}$ with a function $\widehat{f}$ satisfying $\widehat{f}(.4)=.2, \hat{f}(.5)=.2$, and $\widehat{f}(.6)=.4$. While in this example the absolute value of the roundoff error never exceeds 0.05 , not all values up to that bound may be equally likely in practical computations. As Knuth points out in his classic text The Art of Computer Programming [14, pp. 253-255]:

\footnotetext{
In order to analyze the average behavior of floating-point arithmetic algorithms (and in particular to determine their average running time), we need some statistical information that allows us to determine how often various cases arise ... [If, for example, the] leading digits tend to be small [that] makes the most obvious techniques of "average error" estimation for floating-point calculations invalid. The relative error due to rounding is usually ... more than expected.
}

Thus it is an important task to study the distribution of significant digits (or, equivalently, fraction parts of floating-point numbers) for algorithms used in scientific computations. One of the most popular methods in all of applied mathematics is Newton's method, used for computing successive approximations of zeros of functions. The main purpose of this article is to show that Newton's method exhibits exactly the type of nonuniformity of significant digits alluded to by Knuth: not only do the first few significant digits of the distances from the successive approximations to any root, and of the distances between approximations, tend to be small, but-much more specifically - they typically follow a well-known logarithmic (and thus highly nonuniform) distribution called Benford's Law. (Benford's law for significant digits dates back to Newcomb [15]; for overviews of its history and for many empirical and theoretical results, the reader is referred to [13], [16], or [18].)

To formulate Benford's law, recall that for each base (or radix) $b$ in $\mathbb{N} \backslash\{1\}$ every positive real number $x$ is uniquely represented as $x=F_{b} \times b^{e_{b}}$, where $F_{b}=F_{b}(x)$ in $\left[b^{-1}, 1\right)$ is the fraction part of $x$ (base $\left.b\right)$ and $e_{b}=e_{b}(x)$ in $\mathbb{Z}$ is the exponent of $x$ (base $b$ ). (The fraction part $F_{b}(x)$ of $x$ is IEEE Standard terminology, sometimes referred to also as the mantissa or the significand of $x$. It should not be confused with the fractional (or noninteger) part of $x$, namely, $x-\lfloor x\rfloor$, which is independent of the base; here, as usual, $\lfloor x\rfloor$ denotes the largest integer not larger than $x$. For example, the fraction part of $\pi$ base 10 is $.31415 \ldots$, whereas the fractional part of $\pi$ is $.1415 \ldots$.)

A sequence of real numbers $\left(x_{n}\right)=\left(x_{0}, x_{1}, x_{2}, \ldots\right)$ is said to be a Benford sequence base $b$ (or $b$-Benford, for short) if

$$
\lim _{n \rightarrow \infty} \frac{\#\left\{j \leq n: F_{b}\left(\left|x_{j}\right|\right) \leq t\right\}}{n}=1+\log _{b} t \quad\left(t \in\left[b^{-1}, 1\right)\right) .
$$

It is the logarithmic distribution in (1) that is commonly referred to as Benford's law. Its most familiar special case [4], [15] is the decimal form for first significant digits 
base 10 (that is, for the integer part of $\left.10 \cdot F_{10}\right)$, which says that the proportion of $\left(x_{n}\right)$ with leading significant digit $d$ is $\log _{10}\left(1+d^{-1}\right)$ for $d=1,2, \ldots, 9$. In particular, if a sequence of numbers is 10 -Benford, then $\log _{10} 2 \cong 30.1 \%$ of the first significant digits are 1 , and only $\log _{10} \frac{10}{9} \cong 4.58 \%$ are 9 . It is easy to check (see, for example, [10]) that a sequence $\left(x_{n}\right)$ of real numbers is $b$-Benford if and only if

$$
\left(\log _{b}\left|x_{n}\right|\right) \text { is uniformly distributed modulo } 1
$$

(i.e., the limiting proportion of the noninteger parts of $\log _{b}\left|x_{n}\right|$ that lie in the interval $[s, t]$ is $t-s$ whenever $0 \leq s \leq t \leq 1$ ).

Many familiar sequences, such as the Fibonacci numbers and ( $n !)$, are known to be $b$-Benford for all $b$, but it is important to keep in mind that many sequences, including the basic sequence $(n)$ of positive integers, are not. Thus the ubiquitous appearance of Benford's law in Newton's method, as established by the present article, may at first appear surprising. Moreover, as Knuth pointed out, it implies that in algorithms using Newton's method, many methods for estimating the average relative error due to roundoff tend to underestimate the error.

For the most part, these results follow easily from facts about Benford's law in more general dynamical systems [7], and the two main goals here are to specialize those facts to Newton's method, which has a few curious subtleties regarding the multiplicity of roots, and to provide geometrical intuition for the appearance of Benford's law in Newton's method. Since this method is so prevalent in scientific calculations, the results may also help explain the wealth of empirical observations of Benford's law in numerical data (see [4], [11], [13], [16], [18]).

2. NEWTON'S METHOD-THE MAIN THEOREM. Throughout this article, $f: I \rightarrow \mathbb{R}$ is a differentiable function defined on some open interval $I$ in $\mathbb{R}$, and $N_{f}$ denotes its Newton transformation:

$$
N_{f}(x)=x-\frac{f(x)}{f^{\prime}(x)} \quad\left(x \in I \text { with } f^{\prime}(x) \neq 0\right) .
$$

For $N_{f}$ to be defined wherever $f$ is, set $N_{f}(x)=x$ if $f^{\prime}(x)=0$.

Using Newton's method for finding roots (zeros) of $f$ (i.e., real numbers $x^{*}$ with $f\left(x^{*}\right)=0$ ) amounts to picking an initial point $x_{0}$ in $I$ and iterating $N_{f}$. The sequence $\left(x_{n}\right)$ will denote the sequence of iterates of $N_{f}$ starting at $x_{0}$ :

$$
x_{n}=N_{f}\left(x_{n-1}\right)=N_{f}^{n}\left(x_{0}\right) \quad(n \in \mathbb{N}) .
$$

Here and throughout, for any map $T$ of $\mathbb{R}$ (or any subset thereof) into itself $T^{n}$ denotes the $n$-fold composition of $T$ (i.e., $T^{0}(x)=x$ and $T^{n}(x)=T\left(T^{n-1}(x)\right)$ for $n$ in $\mathbb{N}$ ). Note that $N_{f}(x)=x$ if and only if $f(x) f^{\prime}(x)=0$. The intuitive interpretation is that for $x_{n}$ with $f\left(x_{n}\right) f^{\prime}\left(x_{n}\right)=0$ Newton's method terminates at $x_{n}$ : if $f\left(x_{n}\right)=0$, a root has been found; otherwise (3) breaks down due to a horizontal tangent to the graph of $f$ at $x_{n}$ (see Figure 1).

Clearly, if $\left(x_{n}\right)$ converges to $x^{*}$, say, and if $N_{f}$ is continuous at $x^{*}$, then $N_{f}\left(x^{*}\right)=$ $x^{*}$, so $x^{*}$ is a fixed point of $N_{f}$ and $f\left(x^{*}\right)=0$. (According to the definition of $N_{f}$ used here, $N_{f}\left(x^{*}\right)=x^{*}$ could also mean that $f^{\prime}\left(x^{*}\right)=0$. If, however, $f^{\prime}\left(x^{*}\right)=0$ yet $f\left(x^{*}\right) \neq 0$, then $N_{f}$ is not continuous at $x^{*}$ unless $f$ is constant. The analytic properties of $N_{f}$ will be discussed in some detail later.) It is the correspondence between the roots of $f$ and the fixed points of $N_{f}$ that makes Newton's method work locally (see also Figure 1). Under a mild assumption, each fixed point $x^{*}$ is attracting, meaning 


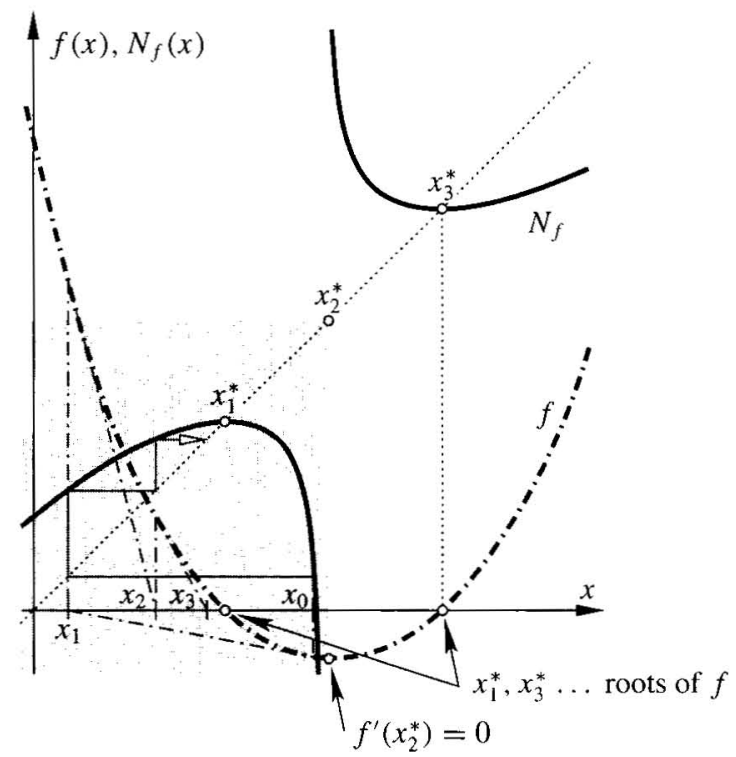

Figure 1. Visualizing Newton's method: The first few iterates $x_{1}, x_{2}$, and $x_{3}$ are found graphically, both by means of the graph of $f$ (broken line) and the graph of $N_{f}$ (solid line). Note how the point $x_{2}^{*}$ with $f^{\prime}\left(x_{2}^{*}\right)=0$ causes $N_{f}$ to have a discontinuity.

that $\lim _{n \rightarrow \infty} N_{f}^{n}\left(x_{0}\right)=x^{*}$ for all $x_{0}$ sufficiently close to $x^{*}$. Although only this local aspect plays a role in the present article, it should be mentioned that for $x_{0}$ further away from any root the sequence $\left(x_{n}\right)$ may exhibit a considerably more complicated long-term behavior. Much attention has been given to this aspect of Newton's method (see for example [3], [5], and [19]).

The main result in this article, Theorem 1 , requires $f$ to be not only differentiable, but even real-analytic. (Recall that a function $f$ is real-analytic if it can be represented by its Taylor's series in a neighborhood of each point in its domain.) Although analyticity is a strong assumption indeed, the class of real-analytic functions covers most practically relevant cases, including all polynomials, all rational, exponential, and trigonometric functions, and compositions thereof [1, chap. 1, Exercise 22]. The reader seeking historical vindication for the restriction to real-analytic functions may recall that one of Newton's objectives in creating this technique was to solve Kepler's equation of celestial mechanics [9]. In modern terminology this means finding roots of the real-analytic function $f(x)=x-\alpha-\beta \sin x$ for positive $\alpha$ and $\beta$. Three centuries later, in Smale's study [19] of Newton's method estimates from data at a single point, the basic assumption throughout the paper is that the underlying functions are analytic.

On the other hand, beyond the class of real-analytic functions (e.g., for functions that are merely infinitely differentiable $\left(C^{\infty}\right)$ ), the distribution of significant digits generated by Newton's method can be practically anything. The reason, in short, is that if $f$ is real-analytic, then its Newton transformation $N_{f}$ is also real-analytic, hence very well-behaved locally, whereas if $f$ is only $C^{\infty}$, then $N_{f}$ may exhibit essentially any pathology imaginable. Some implications of this fact on the conformance of Newton's method to Benford's law (1) will be illustrated by means of examples in section 4 .

If a function is linear in a neighborhood of one of its roots $x^{*}$, then Newton's method terminates after one iteration for every starting point in that neighborhood (i.e., $x_{n}=x^{*}$ for all $n$ in $\mathbb{N}$ ), and nothing interesting can be said about $\left(x_{n}\right)$. In all other 
cases, however, $\left(x_{n}\right)$ typically obeys Benford's law. This is the content of the following theorem, which is the main result in this paper. Recall that $x^{*}$ is a root of multiplicity $k$ of a $C^{\infty}$-function $f$ if $f(x)=\left(x-x^{*}\right)^{k} g(x)$, where $g$ is also of class $C^{\infty}$ and $g\left(x^{*}\right) \neq 0$. A root of multiplicity one is a simple root.

Theorem 1. Suppose that $f: I \rightarrow \mathbb{R}$ is real-analytic, that $f\left(x^{*}\right)=0$, and that $f$ is not linear.

(i) If $x^{*}$ is a simple root of $f$, then $\left(x_{n}-x^{*}\right)$ and $\left(x_{n+1}-x_{n}\right)$ are both $b$-Benford for (Lebesgue) almost all $x_{0}$ in a neighborhood of $x^{*}$ and for all $b$ in $\mathbb{N} \backslash\{1\}$.

(ii) If $x^{*}$ is a double root of $f$, then the same conclusion holds for all $x_{0}$ different from $x^{*}$ in some neighborhood of $x^{*}$, unless $b=2^{j}$ for some $j$ in $\mathbb{N}$.

(iii) If $x^{*}$ is a root of $f$ of multiplicity at least three, then the same conclusion holds for all $x_{0}$ different from $x^{*}$ in some neighborhood of $x^{*}$ and for all $b$ in $\mathbb{N} \backslash\{1\}$.

The proof of Theorem 1 is based on two lemmas. The first establishes the smoothness of the Newton transformation and convergence of the iterates; the second deals with the logarithmic distribution (1) of iterates of more general maps (including, as a special case, the maps associated with Newton's method).

Lemma 1. If $x^{*}$ is a root of multiplicity $k$ of a $C^{\infty}$-function $f: I \rightarrow \mathbb{R}$, then $N_{f}^{\prime}\left(x^{*}\right)=$ $1-k^{-1}$ and, for some open subinterval $J$ of I containing $x^{*}, N_{f}$ belongs to $C^{\infty}(J)$ and $\lim _{n \rightarrow \infty} N_{f}^{n}\left(x_{0}\right)=x^{*}$ for all $x_{0}$ in $J$.

Proof. Since $f(x)=\left(x-x^{*}\right)^{k} g(x)$ for some $g$ in $C^{\infty}(I)$ with $g\left(x^{*}\right) \neq 0$,

$$
N_{f}(x)-x^{*}=\left(x-x^{*}\right) \frac{(k-1) g(x)+\left(x-x^{*}\right) g^{\prime}(x)}{k g(x)+\left(x-x^{*}\right) g^{\prime}(x)}=\left(x-x^{*}\right) h(x),
$$

where $h$ is of class $C^{\infty}$ on some open subinterval $J$ of $I$ containing $x^{*}$ and $N_{f}^{\prime}\left(x^{*}\right)=$ $h\left(x^{*}\right)=1-k^{-1}$. Thus, (4) also shows that

$$
\left|N_{f}(x)-N_{f}\left(x^{*}\right)\right|=\left|N_{f}(x)-x^{*}\right| \leq\left(1-\frac{1}{2 k}\right)\left|x-x^{*}\right|
$$

for all $x$ in $J$, provided that $J$ is chosen sufficiently small. Therefore $N_{f}$ is a contraction on (the closure of) $J$, whence $\lim _{n \rightarrow \infty} N_{f}^{n}\left(x_{0}\right)=x^{*}$ for all $x_{0}$ in $J$.

Note that at any root $x^{*}$ of finite multiplicity of a $C^{\infty}$-function $f$, the derivative of the Newton transformation (i.e., $N_{f}^{\prime}\left(x^{*}\right)$ ) takes its value in the set $\left\{1-j^{-1}: j \in \mathbb{N}\right\}$. For the $C^{\infty}$-function $f(x)=\exp \left(-x^{-2}\right)$, which is not real-analytic at $0, N_{f}^{\prime}(0)=1$. In fact, it can be shown that $N_{f}^{\prime}\left(x^{*}\right)=1$ whenever $N_{f}$ is $C^{1}$ and $x^{*}$ is a root of infinite multiplicity of the $C^{\infty}$-function $f$, i.e., $\lim _{x \rightarrow x^{*}}\left(x-x^{*}\right)^{-n} f(x)=0$ for all $n$ in $\mathbb{N}$. Under a smoothness hypothesis and using the conventions $\mathbb{N}_{\infty}:=\mathbb{N} \cup\{\infty\}$ and $\infty^{-1}:=0$, the property that $T^{\prime}\left(x^{*}\right)$ belongs to $\left\{1-j^{-1}: j \in \mathbb{N}_{\infty}\right\}$ to a large extent characterizes those maps $T$ that are Newton transformations (i.e., $T=N_{f}$ for some $f$; see Theorem 2).

Lemma 2. Suppose that $T$ is in $C^{\infty}(I)$ and that $T\left(y^{*}\right)=y^{*}$ for some $y^{*}$ in $I$.

(a) If $T^{\prime}\left(y^{*}\right) \neq 1$, then for all $b$ in $\mathbb{N} \backslash\{1\}$ and all $y_{0}$ such that $T^{n}\left(y_{0}\right) \rightarrow y^{*}$, the sequence $\left(T^{n}\left(y_{0}\right)-y^{*}\right)$ is b-Benford precisely when $\left(T^{n+1}\left(y_{0}\right)-T^{n}\left(y_{0}\right)\right)$ is b-Benford. 
(b) If $T^{\prime}\left(y^{*}\right)=0$ and $T^{(p)}\left(y^{*}\right) \neq 0$ for some natural number $p>1$, then $\left(T^{n}\left(y_{0}\right)-\right.$ $\left.y^{*}\right)$ is $b$-Benford for all $b$ and (Lebesgue) almost all $y_{0}$ sufficiently close to $y^{*}$.

(c) If $0<\left|T^{\prime}\left(y^{*}\right)\right|<1$, then $\left(T^{n}\left(y_{0}\right)-y^{*}\right)$ is b-Benford for all $y_{0}$ different from but sufficiently close to $y^{*}$ precisely when $\log _{b}\left|T^{\prime}\left(y^{*}\right)\right|$ is irrational.

Proof. For (a), pick $y_{0}$, let $y_{n}=T^{n}\left(y_{0}\right)$, and suppose that $y_{n} \rightarrow y^{*}$. Since $y_{n}=y^{*}$ eventually if and only if $y_{n+1}=y_{n}$ eventually, without loss of generality assume that $y_{n} \neq y^{*}$ for all $n$. Then

$$
y_{n+1}-y_{n}=T\left(y_{n}\right)-y_{n}=\left(y_{n}-y^{*}\right)\left(\frac{T\left(y_{n}\right)-T\left(y^{*}\right)}{y_{n}-y^{*}}-1\right),
$$

so for all $b$ in $\mathbb{N} \backslash\{1\}$

$$
\log _{b}\left|y_{n+1}-y_{n}\right|=\log _{b}\left|y_{n}-y^{*}\right|+\log _{b}\left|\frac{T\left(y_{n}\right)-T\left(y^{*}\right)}{y_{n}-y^{*}}-1\right| .
$$

Since

$$
\lim _{n \rightarrow \infty} \frac{T\left(y_{n}\right)-T\left(y^{*}\right)}{y_{n}-y^{*}}=T^{\prime}\left(y^{*}\right) \neq 1
$$

and since a sequence $\left(z_{n}\right)$ is uniformly distributed mod 1 if and only if $\left(z_{n}+c_{n}\right)$ is uniformly distributed modulo 1 for every convergent sequence $\left(c_{n}\right)$, it follows from (5) that $\left(\log _{b}\left|y_{n}-y^{*}\right|\right)$ is uniformly distributed modulo 1 if and only if $\left(\log _{b}\left|y_{n+1}-y_{n}\right|\right)$ is uniformly distributed modulo 1.

Parts (b) and (c) are the first halves of the conclusions of Theorem 4.1 and Theorem 3.1 of [7], respectively.

Proof of Theorem 1. Let $f$ be real-analytic and not linear, and suppose that $x^{*}$ is a root of $f$. Since the derivative of every real-analytic function is real-analytic [1, Theorem 1.22], $f$ is automatically of class $C^{\infty}$ and $x^{*}$ has finite multiplicity, so by Lemma 1 the iterates $x_{n}=N_{f}^{n}\left(x_{0}\right)$ satisfy $\lim _{n \rightarrow \infty} x_{n}=x^{*}$ for all $x_{0}$ sufficiently close to $x^{*}$. To establish (i), suppose that $x^{*}$ is a simple root of $f$, so $f\left(x^{*}\right)=0$ and $f^{\prime}\left(x^{*}\right) \neq 0$. Since the quotient of real-analytic functions is real-analytic (whenever the denominator is not zero [1, chap. 1, Exercise 22]), the map $N_{f}$ is also real-analytic. By (4), $N_{f}$ satisfies $N_{f}\left(x^{*}\right)=x^{*}, N_{f}^{\prime}\left(x^{*}\right)=0$, and $N_{f}^{(p)}\left(x^{*}\right) \neq 0$ for some integer $p(>1)$. (Otherwise, since $N_{f}$ is real-analytic, $N_{f} \equiv x^{*}$, which implies that $f(x)=c\left(x-x^{*}\right)$ for some constant $c$ and clearly contradicts the hypothesis that $f$ is not linear.) By Lemma 2(b), $\left(x_{n}-x^{*}\right)$ is $b$-Benford for all $b$ in $\mathbb{N} \backslash\{1\}$ and (Lebesgue) almost all $x_{0}$ sufficiently close to $x^{*}$. In view of $\lim _{n \rightarrow \infty} x_{n}=x^{*}$ and Lemma 2(a), this implies that $\left(x_{n+1}-x_{n}\right)$ is also $b$-Benford for all $b$ and almost all $x_{0}$ sufficiently close to $x^{*}$. This proves (i).

To see (ii) and (iii), note that $N_{f}^{\prime}\left(x^{*}\right)=1-k^{-1}$ and $N_{f}^{n}\left(x_{0}\right) \rightarrow x^{*}$ for $x_{0}$ sufficiently close to $x^{*}$ (Lemma 2). By Lemma 2(a),(c) the sequence $\left(x_{n}-x^{*}\right)$ is $b$-Benford if and only if $\log _{b}\left(1-k^{-1}\right)$ is irrational. For a double root (i.e., in the case (ii) corresponding to $k=2$ ) this requires that $b \notin\left\{2^{j}: j \in \mathbb{N}\right\}$. When $k \geq 3$ there is no restriction at all on $b$. (Observe that for $x_{0}$ different from yet sufficiently close to $x^{*}$ Newton's method will not terminate, for otherwise $N_{f} \equiv x^{*}$ and $f$ would be linear.)

In all three cases of Theorem 1, there may be a countably infinite set of starting points $x_{0}$ for which Newton's method terminates, so the sequence $\left(x_{n}\right)$ becomes stationary and in particular is not $b$-Benford for any $b$. The next example illustrates such 
a situation for the single-root case (i); analogues for the cases (ii) and (iii) are given by $f(x)=x^{4}-3 x^{3}+3 x^{2}$ and $f(x)=x^{5}-8 x^{4}+18 x^{3}$, respectively (see also Example 7).

Example 1. Let $f(x)=x^{3}-2 x^{2}+64 x$. Then $f$ has a simple root at $x^{*}=0$, and

$$
N_{f}(x)=\frac{2 x^{3}-2 x^{2}}{3 x^{2}-4 x+64}=-2 x^{2} \frac{1-x}{64-4 x+3 x^{2}} .
$$

It is easy to see that $x^{*}=0$ is the only fixed point of $N_{f}$ and that there exists an increasing sequence $\left(a_{j}\right)=(0,1,4,8, \ldots)$ of real numbers with $N_{f}\left(a_{j+1}\right)=a_{j}$ such that $\lim _{j \rightarrow \infty} a_{j+1} / a_{j}=3 / 2$ and

$$
N_{f}^{-n}(\{0\})=\left\{x_{0}: N_{f}^{j}\left(x_{0}\right)=0 \text { for some } j \leq n\right\}=\left\{a_{1}, a_{2}, \ldots, a_{n}, a_{n+1}\right\} \quad\left(n \in \mathbb{N}_{0}\right) .
$$

Hence, for $x_{0}$ in $\left\{a_{j}: j \in \mathbb{N}\right\}$ Newton's method terminates. If $x_{0} \notin\left\{a_{j}: j \in \mathbb{N}\right\}$, however, Newton's method does not terminate, and for almost all such $x_{0}$ the sequence $\left(x_{n}\right)$ is $b$-Benford for all $b$. The reason for this is that, near zero, $N_{f}(x) \approx-x^{2} / 32$ and Lemma 2(b) applies with $p=2$.

The logarithmic distribution (1) of the iterates of many other root-approximation algorithms follows in the same manner. For instance, one alternative to Newton's method is the one-step Jacobi-Steffensen method [17, p. 65], where the successive approximations $\left(y_{n}\right)$ are defined by

$$
y_{n+1}=y_{n}-\frac{f\left(y_{n}\right)^{2}}{f\left(y_{n}\right)-f\left(y_{n}-f\left(y_{n}\right)\right)} \quad\left(n \in \mathbb{N}_{0}\right) .
$$

In the case of $f(x)=x+x^{3}$, for example, $y_{n}=J_{f}^{n}\left(y_{0}\right)$ with the Jacobi-Steffensen transformation

$$
J_{f}(x)=-x^{5} \frac{1-x^{2}}{1+x^{2}-x^{4}+x^{6}} .
$$

Near zero, $J_{f}(x) \approx-x^{5}$, and Lemma $2(b)$ shows that $\left(y_{n}\right)$ is $b$-Benford for all $b$ and almost all $y_{0}$. Alternatively, $J_{f}=N_{\tilde{f}}$ with the real-analytic function $\tilde{f}(x)=$ $\left(x+x^{3}\right) \exp \left(x^{4} / 4-x^{2}\right)$, hence Theorem 1(i) applies directly as well.

Similarly, results establishing the widespread emergence of Benford sequences for Newton's method in higher dimensions may be inferred from recent more general results for multidimensional dynamical systems [6].

3. GRAPHICAL INTUITION. The purpose of this section is to provide several concrete examples to help the reader visualize how Benford's law appears naturally in floating-point calculations of Newton's method. The first two examples depict functions with simple and double roots, respectively. For illustrative purposes the computations are shown in decimal base (i.e., for $b=10$ ).

Example 2. Let $f(x)=x /(1-x)$ for $x \neq 1$. This function has a simple root at $x^{*}=$ 0 , and the Newton transformation for approximating the root of $f$ is $N_{f}(x)=x^{2}$. Since $F_{10} \circ N_{f} \circ F_{10}=F_{10} \circ N_{f}$, and thus $F_{10}\left(x_{n}\right)=\left(F_{10} \circ N_{f}\right)^{n}\left(x_{0}\right)$ for all $n$, to study the fraction parts $F_{10}\left(x_{n}\right)$ of the floating-point iterations of Newton's method 
near $x^{*}$ it is convenient to study the map $x \mapsto F_{10} \circ N_{f}(x)$ on the interval $[1 / 10,1]$ (see Figure 2 (left)). Note that the decimal fraction part of any number $x$ is the same as the decimal fraction part of $10 x$, so the graph of $F_{10} \circ N_{f}$ consists of two branches: $x \mapsto 10 x^{2}$ on $[1 / 10,1 / \sqrt{10})$ and $x \mapsto x^{2}$ on $[1 / \sqrt{10}, 1]$. Under iteration of the map $F_{10} \circ N_{f}$ on the original scale, meaning for $x$ in $[1 / 10,1]$, almost all orbits are 10Benford. The density corresponding to the logarithmic distribution (1) for $b=10$ is $\pi_{\text {inv }}(x)=(x \log 10)^{-1}$, as shown in Figure 2 (lower left). Note that $\pi_{\mathrm{inv}}$ is the only invariant density of $F_{10} \circ N_{f}$. On the logarithmic scale (that is, for $10^{y-1}=x$ and $y$ in $[0,1]$ ) almost all orbits are uniformly distributed, hence $\pi_{\text {inv }}(y) \equiv 1$ (see Figure 2 (lower right)). Observe that for initial points near zero, although the iterates $x_{n}$ are decreasing to the root $x^{*}=0$, the decimal fraction parts $F_{10}\left(x_{n}\right)$ are not monotonic. For example, if $x_{0}=.5$, then the first three iterates of Newton's method are $.25, .0625$, .00390625 , whose (decimal) fraction parts are $.25, .625$, and .390625 , respectively. Although there is an exceptional set of starting points, which is to say starting points $x_{0}$ such as $1 / 10$ or $1 / \sqrt{10}$ for which $\left(x_{n}\right)$ is not 10 -Benford, the set of such points is small (of Lebesgue measure zero). Since Figure 2 remains the same-up to a relabelling of the axes-if 10 is replaced with any other base, there are no exceptional bases.
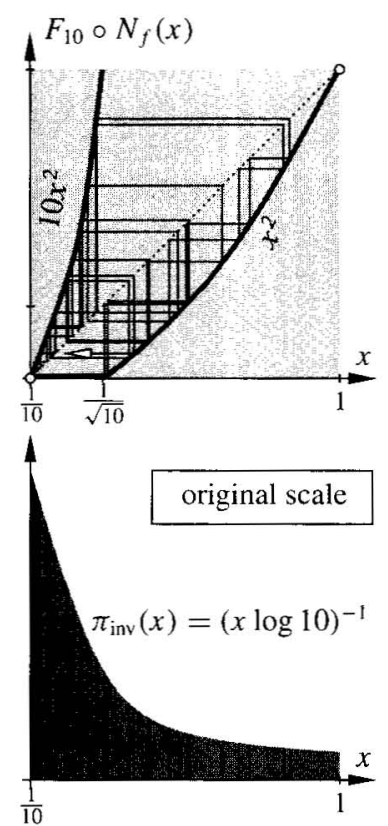
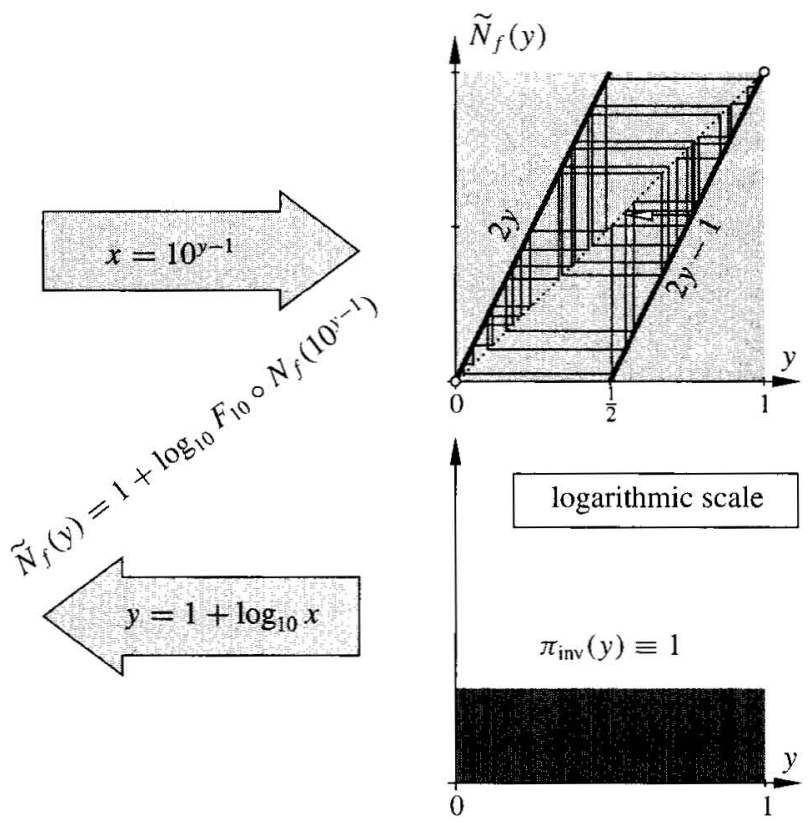

Figure 2. For $f(x)=x /(1-x)$ the associated Newton map is $N_{f}(x)=x^{2}$. On both the original $(x)$ and the logarithmic $(y)$ scale, the first thirty iterates of a typical point $x_{0}$ under $F_{10} \circ N_{f}$ and its logarithmic-scale equivalent $\widetilde{N}_{f}$, respectively, are shown (upper parts). These iterates are also indicated through vertical lines in the plots of the respective invariant densities (lower parts), which gives a clear indication of the fact that the distribution of $F_{10}\left(x_{n}\right)=\left(F_{10} \circ N_{f}\right)^{n}\left(x_{0}\right)$ is skewed towards $1 / 10$, whereas the distribution of $\widetilde{N}_{f}^{n}\left(x_{0}\right)$ is uniform.

Example 3. If $f(x)=x^{2}$, then $f$ has a double root $x^{*}=0$, and $N_{f}(x)=x / 2$. As $F_{10}(x / 2)=F_{10}(5 x)$, the graph of $F_{10} \circ N_{f}$ again consists of two pieces: $x \mapsto 5 x$ on $[1 / 10,1 / 5)$ and $x \mapsto 1 / 2 x$ on $[1 / 5,1]$, as shown in Figure 3 . Unlike the situation in Example 2, the sequence $\left(x_{n}\right)$ of iterates is 10-Benford for all starting points $x_{0}$, but it 
is not $b$-Benford for all bases $b$ : if $b=2^{j}$ for some $j$ in $\mathbb{N}$, then the fraction part base $b$ of $\left(x_{n}\right)$ is $j$-periodic and thus not distributed according to (1). In particular, dividing by $2^{j}$ (i.e., applying $N_{f} j$ times) shifts the $b$-digits one place to the right but leaves the fraction part unchanged.
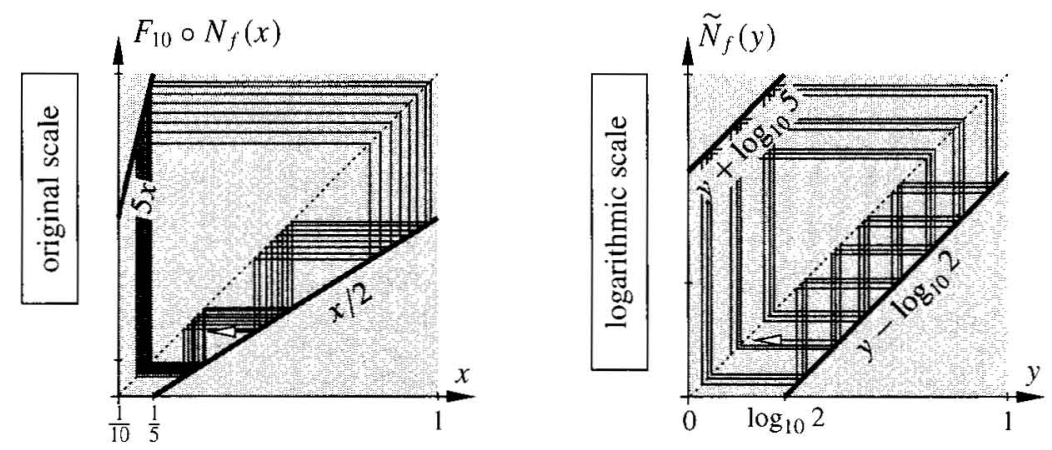

Figure 3. Newton's method for the double root $x^{*}=0$ of $f(x)=x^{2}$, for which $N_{f}(x)=x / 2$. Again the system is most easily understood on the logarithmic scale, as $\widetilde{N}_{f}$ is a piecewise isometry (see Example 2 and Figure 2 for notations).

Examples 2 and 3 have been chosen so that their Newton transformations are monomials. In the nonmonomial case, $N_{f}$ cannot be turned into a self-map of a compact interval in a natural way. For example, for a function with simple root at 0 (e.g., $g(x)=$ $x+x^{2}$, whose Newton transformation is $\left.N_{g}(x)=x^{2} /(1+2 x)\right)$ the graphs are not as neat. In this case, the limiting distribution of the fraction part is obtained indirectly, via the monomial case, using a technique of "shadowing." Since $x^{2} /(1+2 x) \approx x^{2}$ for $x$ close to 0 , shadowing implies that the asymptotics of the fraction parts of the iterates of both functions are identical near 0 . The idea of shadowing is that, even though

$$
\left|\log _{b} N_{g}^{n}(x)-\log _{b} N_{f}^{n}(x)\right| \rightarrow \infty
$$

as $n \rightarrow \infty$, for each $x$ near 0 there exists precisely one number $s=s(x)$, the shadow of $x$, with

$$
\left|\log _{b} N_{g}^{n}(s)-\log _{b} N_{f}^{n}(x)\right| \rightarrow 0
$$

such that both $N_{g}$ and $N_{f}$ generate the same asymptotics (see [7] for details). For example, unlike in the case

$$
f(x)=x /(1-x)=x+x^{2}+x^{3}+\cdots
$$

where $N_{f}(x)=x^{2}$, Newton's method for $g(x)=x+x^{2}$ does not induce a map of the interval $[1 / 10,1]$ into itself, because $F_{10} \circ N_{g} \circ F_{10} \neq F_{10} \circ N_{g}$. Nevertheless, shadowing implies that $\left(N_{f}^{n}(x)\right)$ and $\left(N_{g}^{n}(x)\right)$ have essentially the same asymptotics of significant digits.

4. SMOOTH FUNCTIONS MAY CAUSE PATHOLOGIES. Ideally, it would be desirable to extend the conclusion of Theorem 1 to a wider class of functions (e.g., to all $C^{\infty}$-functions). For this it is imperative that $N_{f}$ be analytically well-behaved whenever $f$ is. However, it is a simple fact, hardly ever alluded to in studies of Newton's method, that the analytic properties of $f$ and $N_{f}$ are quite independent. On the one 
hand, the $C^{1}$-function $f(x)=|x|^{3 / 2}$, which is not of class $C^{2}$, has a $C^{\infty}$-function as its Newton transformation, namely, $N_{f}(x)=x / 3$. On the other hand, $N_{f}$ may lack decent analytic properties even if $f$ is smooth, as the following example shows.

Example 4. It is easily seen that the function $f: \mathbb{R} \rightarrow \mathbb{R}$ defined by

$$
f(x)= \begin{cases}\exp \left(-x^{-2}+|x|+\cos \left(x^{-2}\right)\right) & \text { if } x \neq 0, \\ 0 & \text { if } x=0\end{cases}
$$

is in $C^{\infty}(\mathbb{R})$ and that both $f$ and $f^{\prime}$ vanish only at $x^{*}=0$. Nevertheless

$$
-1=\liminf _{x \rightarrow 0} N_{f}(x)<\limsup _{x \rightarrow 0} N_{f}(x)=1,
$$

hence $N_{f}$ is not even continuous at $x^{*}$.

Since $N_{f}$ may fail to be continuous even if $f$ is a $C^{\infty}$-function, some assumption on the smoothness of $N_{f}$ has to be imposed explicitly. Otherwise Newton's method may not be applicable at all, and as a consequence - and more to the point - the fraction parts of $\left(x_{n}\right)$ cannot reasonably be expected to follow any particular distribution. An additional smoothness assumption on $N_{f}$ in turn raises the question of whether such a condition can be met at all for a reasonably large class of functions and, if so, exactly which maps arise that way. The next theorem provides a complete answer to this question in the setting where both the function and its Newton transformation are infinitely differentiable: It characterizes those $C^{\infty}$-maps $T$ that satisfy $T=N_{f}$ for some $f$ of class $C^{\infty}$. Naturally, this characterization clarifies which functions any potential extension of Theorem 1 would have to cover (see also Figure 4).
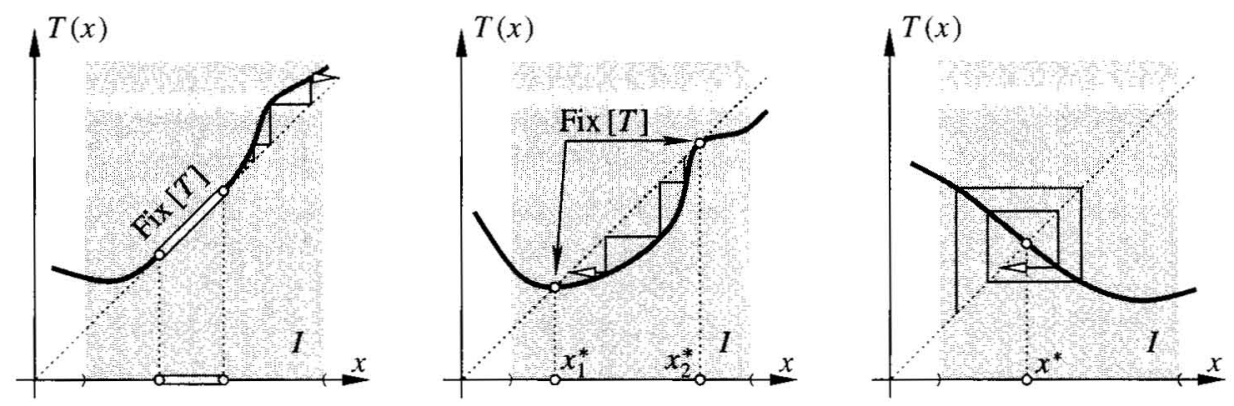

Figure 4. Three $C^{\infty}$-maps $T$ that are not Newton transformations associated with any $C^{\infty}$-function on the interval $I$, because Fix $[T]$ is not attracting (left), Fix $[T]$ is not an interval (middle), and $T^{\prime}\left(x^{*}\right) \notin\left\{1-j^{-1}\right.$ : $\left.j \in \mathbb{N}_{\infty}\right\}$, respectively.

For any map $T: I \rightarrow I$ let Fix [T] signify the set of fixed points of $T$ (that is, Fix $[T]:=\{x \in I: T(x)=x\})$. The set Fix [T] is attracting if $\lim _{n \rightarrow \infty} T^{n}\left(x_{0}\right)$ belongs to Fix $[T]$ for all $x_{0}$ sufficiently close to Fix [T].

Theorem 2. Suppose that $T$ belongs to $C^{\infty}(I)$. Then $T=N_{f}$ for some $f$ in $C^{\infty}(I)$ if and only if Fix $[T]$ is either empty or an attracting (possibly one-point) interval, and

$$
T^{\prime}(\text { Fix }[T])=\left\{1-k^{-1}\right\}
$$

for some $k$ in $\mathbb{N}_{\infty}$. Moreover, $f$ is uniquely determined up to a multiplicative constant if either $k \neq \infty$ in (6) or the set $I \backslash$ Fix $[T]$ is connected. 
From Lemma 1 and the discussion thereafter, it is quite clear that condition (6) is necessary for $T$ to be the Newton transformation associated with a $C^{\infty}$-function. To see that (6) is also sufficient is slightly harder and requires an in-depth analysis of the differential equation $N_{f}=T$ or, equivalently, $f^{\prime}(x)=(x-T(x))^{-1} f(x)$. The underlying intuition is that, in the context of Newton's method, smooth maps and functions are essentially determined by their leading terms. Since Theorem 2 will be used only rather informally in what follows, no proof is given here. The interested reader is referred to [8] for a proof and further details.

As discussed earlier, Theorem 2 and its consequences have an immediate bearing on the generation of Benford sequences under Newton's method. By Example 4, extending Theorem 1 to smooth functions would require either explicitly postulating that $N_{f}$ be at least of class $C^{1}$ or excluding roots of infinite multiplicity (see [8]). Even disregarding this, there is no hope of extending Theorem 1 to $C^{\infty}$-functions, as will be demonstrated now by means of three examples that address potential pathologies in the case of a root of infinite multiplicity and the case of a very degenerate simple root, respectively.

Example 5. The function $f: \mathbb{R} \rightarrow \mathbb{R}$ defined by $f(x)=\exp \left(-x^{-2}\right)$ if $x \neq 0$ and $f(0)=0$ has $x^{*}=0$ as a root of infinite multiplicity, and $N_{f}(x)=x\left(1-x^{2} / 2\right)$ satisfies $N_{f}^{\prime}\left(x^{*}\right)=1$. By [7, Theorem 3.4], since $N_{f} \in C^{\infty}(\mathbb{R})$, the sequence $\left(x_{n}\right)$ is not $b$-Benford for any $b$ whenever $\lim _{n \rightarrow \infty} x_{n}=0$. (Note that this is the case if and only if $\left|x_{0}\right|<2$; when $\left|x_{0}\right|>2$ a "reciprocal" version of Lemma 2(b) applies, and $\left(x_{n}\right)$ is typically $b$-Benford for all $b$.)

On the other hand, consider the $C^{\infty}$-function $g: \mathbb{R} \rightarrow \mathbb{R}$ given by

$$
g(x)= \begin{cases}\exp \left(\frac{3}{2} x^{2}-\frac{1}{4}(\log |x|)^{4}\right) & \text { if } x \neq 0 \\ 0 & \text { if } x=0\end{cases}
$$

for which $N_{g}(0)=0$ and

$$
N_{g}(x)=x\left(1-\left(3 x^{2}-(\log |x|)^{3}\right)^{-1}\right) \quad(x \neq 0) .
$$

Clearly $N_{g}^{\prime}(0)=1$, yet $N_{g}$ is merely in $C^{1}(\mathbb{R})$. As in [7, Example 3.5], the sequence $\left(x_{n}\right)$ is $b$-Benford for all $b$ whenever $x_{0} \neq 0$.

Thus, near a root of infinite multiplicity of a $C^{\infty}$-function $f$, the generation of Benford sequences may depend critically on the smoothness of $N_{f}$, which-as evidenced by Example 4-is hard to control from the outset.

Example 6. By Theorem 2, every $C^{\infty}$-map $T$ with $T\left(x^{*}\right)=x^{*}$ and $T^{(j)}\left(x^{*}\right)=0$ for all positive $j$ but with $T(x) \neq x$ whenever $x \neq x^{*}$, satisfies $T=N_{f}$ near $x^{*}$ for some $C^{\infty}$-function $f$. It follows from [7, Example 4.7] that near such a flat super-attracting fixed point a general statement (positive or negative) concerning the generation of Benford sequences cannot possibly be made.

Concretely, assume that $\varphi$ in $C^{\infty}(\mathbb{R})$ satisfies $\varphi^{(j)}(0)=0$ for all $j$ in $\mathbb{N}_{0}$, yet $\varphi(x)>$ 0 when $x \neq 0$. Then the function $f: \mathbb{R} \rightarrow \mathbb{R}$ given by $f: x \mapsto x(1+\varphi(x))$ has $x^{*}=0$ as a simple root, and

$$
N_{f}(x)=\frac{x^{2} \varphi^{\prime}(x)}{1+\varphi(x)+x \varphi^{\prime}(x)} .
$$

Thus $N_{f}^{(j)}(0)=0$ for all $j$ in $\mathbb{N}_{0}$, and $\left(x_{n}\right)$ may or may not be a Benford sequence. For example, if $\varphi(x)=\exp \left(-x^{-2}\right)$ then $\left(x_{n}\right)$ is $b$-Benford for almost all $x_{0}$ and all $b$, as 
follows easily from [7, Theorem 4.4]. On the other hand, from [7, Example 4.7] it is straightforward to find functions $\varphi$ such that Newton's method does not produce any Benford sequence at all.

Example 7. Finally, it is important to keep in mind that Newton's method may terminate (i.e., a root may be reached after only a finite number of iteration steps), as was seen in Example 1. More generally, if $x^{*}$ is a root of $f: I \rightarrow \mathbb{R}$ and $N_{f}$ belongs to $C^{1}(I)$, let $\Omega_{f}$ denote the set of points $x_{0}$ in $I$ for which $\left(x_{n}\right)$ terminates at $x^{*}$ :

$$
\Omega_{f}=\left\{x_{0} \in I: N_{f}^{j}\left(x_{0}\right)=x^{*} \text { for some } j \in \mathbb{N}\right\} .
$$

Clearly, in terms of Benford's law nothing interesting can be said about Newton's method starting at points in $\Omega_{f}$. In Example $1, \Omega_{f}$ is countable and discrete. In particular, $x^{*}=0$ has a positive distance from $\Omega_{f} \backslash\left\{x^{*}\right\}$. This is a manifestation of the general fact that, for real-analytic $f, \Omega_{f}$ cannot accumulate anywhere within the domain of attraction of $x^{*}$ (i.e., within the open set $\left\{x_{0} \in I: \lim _{n \rightarrow \infty} N_{f}^{n}\left(x_{0}\right)=x^{*}\right\}$ ). In Example 1 the domain of attraction of $x^{*}=0$ is $\mathbb{R}$. In stark contrast, however, it is fairly easy to produce a $C^{\infty}$-function $f$ with $f(0)=0$ that is not linear on any interval but has $\Omega_{f}$ much larger (in particular, $\Omega_{f}$ has $x^{*}=0$ as a (Lebesgue) density point).

To exhibit such a function $f$, first construct a family of subintervals $I_{j}^{(n)}$ of $[0,1]$ for $n=1,2, \ldots$ and $j=1, \ldots, 2^{n-1}$ inductively as follows: The interval $I_{1}^{(1)}$ is centered at $1 / 2$ with length $1 / 4$ (i.e., $I_{1}^{(1)}=[3 / 8,5 / 8]$ ); the intervals $I_{1}^{(2)}$ and $I_{2}^{(2)}$ are centered in the two pieces of $C_{1}:=\overline{[0,1] \backslash I_{1}^{(1)}}$ and both have length $1 / 16$. At the $(n+1)$ th step, the $2^{n}$ intervals $I_{j}^{(n+1)}$ are centered within the $2^{n}$ pieces of $C_{n}:=\overline{[0,1] \backslash \bigcup_{k=1}^{n} \bigcup_{j=1}^{2^{k-1}} I_{j}^{(k)}}$, and all have equal length $4^{-(n+1)}$. Write $I_{j}^{(n)}=\left[a_{j}^{(n)}, b_{j}^{(n)}\right]$, and note that the total length of $C_{n}$ is $1 / 2+2^{-(n+1)}$. The set $C:=\bigcap_{n=1}^{\infty} C_{n}$, a "fat" Cantor set, is compact and nowhere dense with Lebesgue measure $\lambda(C)=1 / 2$. It is easy to check that $\delta^{-1} \lambda(C \cap$ $[0, \delta]) \rightarrow 1$ as $\delta \searrow 0$.

Let $\psi$ denote the $C^{\infty}$-function defined on the real line by

$$
\psi(x):= \begin{cases}\exp \left(4-x^{-1}-(1-x)^{-1}\right) & \text { if } 0<x<1 \\ 0 & \text { otherwise }\end{cases}
$$

and define a sequence $\left(\psi_{n}\right)$ of nonnegative functions on $\mathbb{R}$ by

$$
\psi_{n}(x)=\exp \left(-4^{n}\right) \sum_{j=1}^{2^{n-1}} \psi\left(4^{n}\left(x-a_{j}^{(n)}\right)\right) \quad(n \in \mathbb{N}) .
$$

Clearly, each $\psi_{n}$ is in $C^{\infty}(\mathbb{R})$ and, together with all its derivatives, vanishes everywhere except on $\bigcup_{j=1}^{2^{n-1}} I_{j}^{(n)}$. In particular, $\psi_{n}$ and $\psi_{m}$ have disjoint supports when $n \neq m$. Moreover, for each $k$ in $\mathbb{N}_{0}$ there exists a constant $\gamma_{k}>0$ such that $\left|\psi_{n}^{(k)}(x)\right| \leq \gamma_{k}$ for all $n$ and $x$; specifically, one can choose $\gamma_{0}=\gamma_{1}=1$. Consequently,

$$
\varphi(x):=\exp (-\lfloor|x|\rfloor) \sum_{n=1}^{\infty} \psi_{n}(x-\lfloor x\rfloor) \quad(x \in \mathbb{R})
$$

defines a $C^{\infty}$-function with $0 \leq \varphi(x)<\exp (1-|x|)$ and $\left|\varphi^{\prime}(x)\right|<\exp (1-|x|)$. Observe that $\varphi$ vanishes exactly on $C+\mathbb{Z}=\{c+k: c \in C, k \in \mathbb{Z}\}$. As in Example 6, use $\varphi$ to define $f(x)=x(1+\varphi(x))$ and notice that $f$ is not linear on any interval. Obviously, $f$ has a unique, simple root at $x^{*}=0$. The associated Newton map $N_{f}$ is 
itself of class $C^{\infty}$, as is obvious from (7), and it vanishes on a set containing $C+\mathbb{Z}$, whence $\Omega_{f}$ contains $C+\mathbb{Z}$. In this example, not only does $\Omega_{f}$ have positive measure but, even worse,

$$
\frac{\lambda\left(\Omega_{f} \cap[-\delta, \delta]\right)}{2 \delta} \rightarrow 1
$$

as $\delta \searrow 0$. This means that close to $x^{*}=0$ truncation of Newton's method (in fact, after merely one iteration step) is the rule-an unpleasant fact obviously precluding any reasonable statement about Benford's law in this case!

5. CONCLUSIONS. With the prevalence of Benford's law under Newton's method established for the case when the iterates converge to a root, the case most relevant in practical computations, it seems fitting that this article close by highlighting a few implications of Benford's law for scientific computing. By Theorem 1 and its many variants, these implications pertain to the myriad numerical algorithms based on Newton's method, as well as to many other settings in both deterministic and stochastic frameworks (see [7], [13]).

The appearance of Benford's law in scientific computing is a well-known empirical fact [2], [11], [14], [18]. Hamming gives "a number of applications to hardware, software, and general computing which show that this distribution is not merely an amusing curiosity" [12, p. 1609]. Schatte analyzes the speed of multiplication and division in digital computers when the statistical distribution of floating-point numbers is logarithmic and proves that, for design of computers, "The base $b=2^{3}$ is optimal with respect to [minimizing expected] storage use" [18, p. 453].

The prevalence of Benford's law has important practical implications for (digital) computers that use floating-point arithmetic, and the errors inherent in these devices. One type of error in scientific calculations is overflow (or underflow), which occurs when the running calculations exceed the largest (or smallest, in absolute value) floating-point number allowed by the computer. Feldstein and Turner show that $[\mathbf{1 1}$, p. 241]

[u]nder the assumption of the logarithmic distribution of numbers [i.e., Benford's Law], floating-point addition and subtraction can result in overflow and underflow with alarming frequency.... .

Together with Theorem 1, this suggests that overflow and underflow errors should be given careful attention by any computer algorithm using Newton's method.

Another important type of error in scientific computing arises due to roundoff. In fact, justified by heuristics and by the extensive empirical evidence of Benford's law in numerical calculations, analysis of roundoff errors has often been carried out under the hypothesis of a logarithmic statistical distribution. For example, in their study of roundoff errors, Barlow and Bareiss state that [2, p. 326] "For all standard operations we assume that $x$ approximately follows the reciprocal distribution. ..."-that is, Benford's law, whose density is the reciprocal function $(x \log b)^{-1}$, as seen in Figure 2 for the case $b=10$.

As Knuth pointed out, an assumption of uniformly distributed fraction parts in calculations using floating-point arithmetic tends to underestimate the average relative roundoff error in cases where the actual statistical distribution of fraction parts is skewed toward smaller leading significant digits (see the introductory quote from [14]). To obtain a rough idea of the magnitude of this underestimate when the true statistical distribution is Benford's law, consider the case of decimal floating-point arithmetic. 
Let $X$ denote the absolute roundoff error at the time of stopping the algorithm, and let $Y$ denote the fraction part of the approximation at the time of stopping. Thus the relative error is $X / Y$, and assuming that $X$ and $Y$ are independent random variables, the average (i.e., expected) relative error is simply $E X \cdot E(1 / Y)$. If $Y$ is assumed to be uniformly distributed on $[1 / 10,1)$, then the expected average relative error is

$$
\int_{0.1}^{1} \frac{10 d t}{9 t}=\frac{10 \log 10}{9} \approx 2.558
$$

times the expected absolute error $E X$, whereas if the true distribution of $Y$ is Benford, then the true average relative error is

$$
\int_{0.1}^{1} \frac{d t}{t^{2} \log 10}=\frac{9}{\log 10} \approx 3.909
$$

times the expected absolute error. Thus, for example, in numerical algorithms based on Newton's method, ignoring the fact that $Y$ is Benford creates an average underestimation of the relative error by more than one third!

ACKNOWLEDGMENTS. The first author was partially supported by a Humboldt fellowship. He wishes to thank the Johann Wolfgang von Goethe-Universität Frankfurt for its hospitality. The second author was partially supported by a National Security Agency Grant. He is grateful to the University of Leiden and the Universität Bonn for invitations to visit in April 2005, during which time some of this research was undertaken, and to the California Polytechnic State University for its hospitality and its support as Research Scholar in Residence 2005-2006. Both authors are thankful to an anonymous referee for several corrections and suggestions that improved the exposition.

\section{REFERENCES}

1. S. Axler, P. Bourdon, and W. Ramey, Harmonic Function Theory, Springer-Verlag, New York, 1992.

2. J. Barlow and E. Bareiss, On roundoff error distributions and floating-point and logarithmic arithmetic, Computing 34 (1985) 325-347.

3. A. Beardon, Iteration of Rational Functions, Springer-Verlag, New York, 1991.

4. F. Benford, The law of anomalous numbers, Proc. Amer. Philosophical Soc. 78 (1938) 551-572.

5. A. Berger, Chaos and Chance, deGruyter, Berlin, 2001.

6. - Multi-dimensional dynamical systems and Benford's law. Discrete Contin. Dyn. Syst. Ser. A 13 (2005) 217-237.

7. A. Berger, L. Bunimovich, and T. Hill, One-dimensional dynamical systems and Benford's law, Trans. Amer. Math. Soc. 357 (2005) 197-219.

8. A. Berger and T. Hill, A characterisation of Newton maps, ANZIAM J. 48 (2006) 211-223.

9. P. Colwell, Solving Kepler's Equation Over Three Centuries, Willmann-Bell, Richmond VA, 1993.

10. P. Diaconis, The distribution of leading digits and uniform distribution mod 1, Ann. Probab. 5 (1977) 72-81.

11. A. Feldstein and P. Turner, Overflow, underflow, and severe loss of significance in floating-point addition and subtraction, IMA J. Numerical Anal. 6 (1986) 241-251.

12. R. Hamming, On the distribution of numbers, Bell System Technical Journal 49 (1970) 1609-1625.

13. T. Hill, A statistical derivation of the significant-digit law, Statistical Science 10 (1996) 354-363.

14. D. Knuth, The Art of Computer Programming, vol. 2, 3rd ed., Addison-Wesley, Reading, MA, 1997.

15. S. Newcomb, Note on the frequency of use of the different digits in natural numbers, Amer. J. Math. 4 (1881) 39-40.

16. R. Raimi, The first digit problem, this MONTHLY 102 (1976) 322-327.

17. W. Rheinboldt, Methods for Solving Systems of Nonlinear Equations, 2nd ed., SIAM, Arrowsmith, Bristol, UK, 1998.

18. P. Schatte, On mantissa distributions in computing and Benford's law, J. Inform. Process. Cybernet. 24 (1988) $443-455$.

19. S. Smale, Newton's method estimates from data at one point, in The Merging of Disciplines: New Directions in Pure, Applied and Computational Mathematics, Springer-Verlag, New York, 1986, pp.185-196. 
ARNO BERGER has been intrigued by dynamical systems ever since he had the privilege of learning about the subject from some illustrious people. A native of Austria, he spent extended research visits to the U.S., U.K., and recently Germany. Since 2004 he finds himself almost antipodal to his native country yet with an inspiring view on some other mountains, aptly named the Southern Alps.

Department of Mathematics and Statistics, University of Canterbury, Christchurch, New Zealand arno.berger@canterbury.ac.nz

TED HILL is professor emeritus of mathematics at Georgia Tech and currently research scholar in residence at the California Polytechnic State University in San Luis Obispo, where his view overlooking Morro Bay is also inspiring. He has held academic positions in Germany, Mexico, Hawaii, Holland, Costa Rica, and Israel, and recently donated his collection of a thousand early American mathematics books to Bancroft Library's rare books archives at UC Berkeley, where as a graduate student three decades ago, he first learned about Benford's law in a colloquium lecture by Lester Dubins.

School of Mathematics, Georgia Institute of Technology, Atlanta GA 30332-0160 USA

hill@math.gatech.edu 